\title{
Modeling of the Sampling Effect in the P-Type Average Current Mode Control
}

\author{
Young-Seok Jung ${ }^{\dagger}$ and Marn-Go Kim* \\ $\dagger^{*}$ Dept. of Mechanical and Automotive Eng., Pukyong National University, Busan, Korea
}

\begin{abstract}
This paper presents the modeling of the sampling effect in the p-type average current mode control. The prediction of the high frequency components near half of the switching frequency in the current loop gain is given for the p-type average current mode control. By the proposed model, the prediction accuracy is improved when compared to that of conventional models. The proposed method is applied to a buck converter, and then the measurement results are analyzed.
\end{abstract}

Key Words: Average current mode control, P-type controller, Sampling effect, sampler model

\section{INTRODUCTION}

Average current mode control was developed in the early 90's [1] and has been widely used in many converter applications where a low harmonic content of the converter input current is necessary [2]-[4]. Average current mode control has several advantages when compared with peak current mode control such as its not needing an external stabilizing ramp signal, the high current loop gain in the low frequency region, and an improved noise immunity. Particularly, in a power factor correction applications, the average current mode control can lower the distortion of the input current when compared with the peak current mode control.

An accurate dynamic model is necessary for the effective design of PWM converters. For this reason, many technical papers dealing with the modeling and design of average current mode control have been reported [2]-[4]. The small signal model considering the sampling effect in a current control loop has been known as an accurate one particularly in peak current mode control [5], [6]. It has also been proven in [7] that the consideration of the sampling effect can improve the prediction accuracy of average current mode control employing proportional and integral type (PI-type) controllers. In many previous works, the possibility of current loop instability with PI-type average current mode control has also been mentioned. One way of avoiding this instability problem is to use P-type control which is widely used in many industrial applications due to its simplicity.

In this paper, we intend to show that the accuracy improvement by the previous sampler model obtained for PItype average current mode control is limited in its application

Manuscript received Jul. 23, 2010; revised Nov. 15, 2010

$\dagger$ Corresponding Author: yousjung@ @ pknu.ac.kr

Tel: +82-51-629-6165, Fax: +82-51-629-6150, PKNU

* Dept. of Control and Instrumentation Eng., Pukyong National University, Korea to P-type control. To solve this problem, we will modify the sampler model and verify that the modified model will improve the prediction accuracy.

This paper is organized as follows. The sampler model for PI-type average current mode control is reviewed in Section II. Problem statements for the direct utilization of the previous sampler model are presented in Section III. The proposed sampler model for the P-type sampler model is illustrated in Section IV. Validations and some conclusions are given in Section V and VI, respectively.

\section{SAmpler Model for PI-type Average Current MODE CONTROL}

Fig. 1 shows a PWM converter with PI-type average current mode control. In this figure, a power stage, a current compensator and a PWM modulator are shown. The inductor current is sensed through a current sensor whose gain is $R_{s}$. The current compensator has two poles and one zero and its transfer function can be written as

$$
H_{c}(s)=\frac{K_{c}\left(1+s / \omega_{z}\right)}{s\left(1+s / \omega_{p}\right)}
$$

where the DC gain, $K_{c}$, the high-frequency pole, $\omega_{p}$, and the zero, $\omega_{z}$, are defined by

$$
K_{c}=\frac{1}{R_{l}\left(C_{p}+C_{z}\right)}, \omega_{z}=\frac{1}{R_{f} C_{z}}, \omega_{p}=\frac{C_{z}+C_{p}}{R_{f} C_{z} C_{p}} .
$$

In order to obtain a complete small signal model for the converter system, the modeling for the modulator and the power stage are conducted separately and then combined with the model of the current compensator to obtain the final model. Fig. 2 shows the small signal model structure of PI-type average current mode control. The gains, $K_{f}$ and $K_{r}$, are related to the input and output voltages, but, in this paper, we are not concerned with these gains because their effect should be negligible in counting the sampling effect in the current loop. 


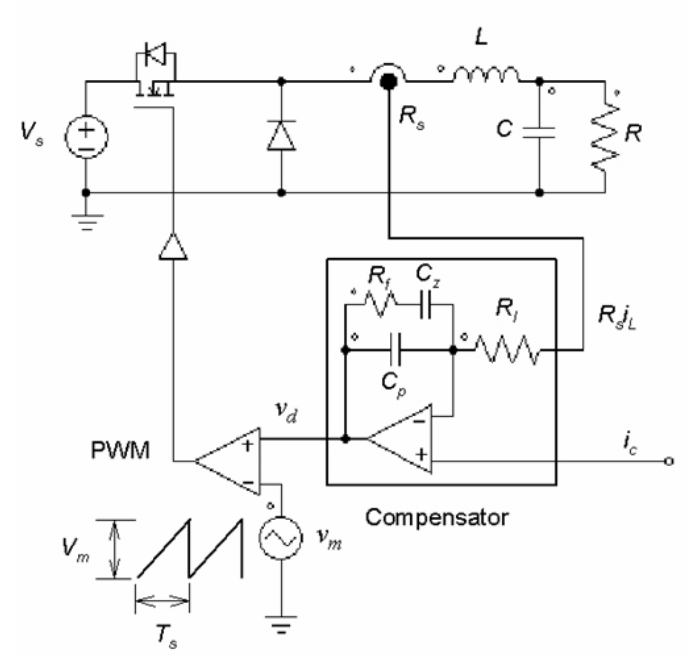

Fig. 1. The PWM converter with PI-type average current mode control.

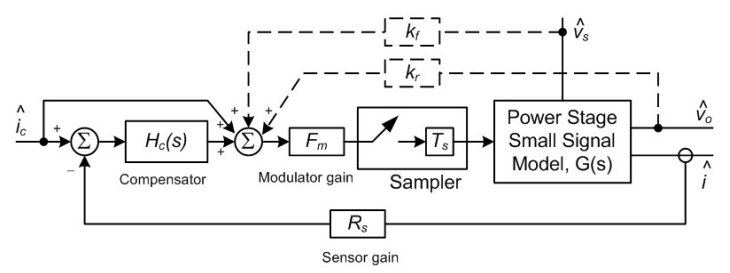

Fig. 2. Small signal model structure of the PI-type average current mode control.

The gain blocks shown in Fig. 2 can be easily obtained as follows [7]:

$$
\begin{gathered}
G(s)=\frac{M_{r}+M_{f}}{s} \\
F_{m}=\frac{\hat{d}}{\hat{v}_{d}}=\frac{1}{\left(M_{c}+M_{1}\right) T_{s}}
\end{gathered}
$$

where, $M_{r}$ and $M_{f}$ represent the rising and falling slopes of the inductor current, respectively,

$$
\begin{aligned}
M_{1}= & k_{3}+k_{4}+\frac{k_{1}-k_{2}+\left(k_{3}+k_{4}\right) e^{-\omega_{p}(1-D) T_{s}}}{1-e^{-\omega_{p} T_{s}}} e^{-\omega_{p} D T_{s}} \\
k_{1} & =R_{s} K_{c}\left(\frac{I_{p p}}{2}-\frac{M_{f}}{\omega_{z}}\right)\left(1-e^{-\omega_{p}(1-D) T_{s}}\right) \\
& k_{2}=\frac{R_{s} K_{c} M_{f}}{\omega_{p}}\left(\omega_{p}(1-D) T_{s}-1+e^{-\omega_{p}(1-D) T_{s}}\right) \\
& k_{3}=R_{s} K_{c}\left(\frac{M_{r}}{\omega_{z}}-\frac{I_{p p}}{2}\right)\left(1-e^{-\omega_{p} D T_{s}}\right) \\
& k_{4}=\frac{R_{s} K_{c} M_{r}}{\omega_{p}}\left(\omega_{p} D T_{s}-1+e^{-\omega_{p} D T_{s}}\right)
\end{aligned}
$$

and $I_{P P}$ is the peak to peak inductor current ripple. Detailed derivations of the above equations can be found in [7]. Table 1 shows the rising and falling slopes for three basic converters. From the modeling structure shown in Fig. 2 and some manipulations of the transfer function, a sampler model can be obtained as in previous works for PI-type average current mode control as

$$
H_{s}(s)=\frac{1}{1+R_{s} F_{m} T_{s}\left[H_{c}(s) G(s)\right]^{*}-R_{s} F_{m} H_{c}(s) G(s)}
$$

where the upper asterisk is used for the sampled quantity. In the following section, we will discuss the inaccuracy of
TABLE I

RISING AND FALLING SLOPES FOR THREE BASIC CONVERTER

\begin{tabular}{|c|c|c|c|}
\hline & Rising slope, & Falling slope, & $M_{r}+M_{f}$ \\
\hline Boost & $M_{r}$ & $M_{f}$ & \\
Buck & $V_{s} / L$ & $\left(V_{o}-V_{s}\right) / L$ & $V_{o} / L$ \\
Buck-Boost & $\left(V_{s}-V_{o}\right) / L$ & $V_{o} / L$ & $V_{s} / L$ \\
$V_{s} / L$ & $V_{o} / L$ & $\left(V_{s}+V_{o}\right) / L$ \\
\hline
\end{tabular}

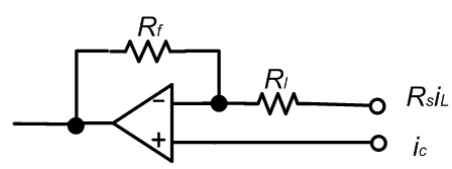

Fig. 3. P-type compensator.

the direct application of this sampler model to P-type average current mode control.

\section{Problem Statements}

For PI-type control, the accuracy of the sampler model expressed in (5) has been proven in previous works. In order to apply the sampler model to P-type average current mode control, a redefinition and/or re-calculation of the modulator and compensator gains are required. P-type average current mode control can be constructed by replacing the PI-type compensator with a P-type one. In Fig. 3, a P-type compensator is shown.

From Fig. 3, the transfer function of P-type compensator can be simply derived as

$$
H_{c}(s)=K_{c}=\frac{R_{f}}{R_{l}} .
$$

In Fig. 4, the waveforms of the PWM modulator are shown. The compensator output $v_{d}$ is compared with the triangular waveform, and a PWM signal for the power switch is generated. From this figure, the modulator gain can be calculated as

$$
F_{m}=\frac{\hat{d}}{\hat{v}_{d}}=\frac{1}{\left(M_{c}+R_{s} K_{c} M_{r}\right) T_{s}} .
$$

The examining converter parameters are listed in Table 2. Using the converter parameters, the frequency responses of the loop gain are tested. In Fig. 5, the results from the analysis utilizing equation (5) and a switching level simulation, in this study we use PSIM, are presented.

From this figure, the inaccuracy of the conventional model is observed. Particularly, the magnitude of the conventional model is lower than the prediction results from the PSIM and the phase delay near half of the switching frequency is also less than it is in the PSIM results.

TABLE II

PARAMETERS OF EXAMPLE CONVERTER

\begin{tabular}{|l|l|}
\hline Operating PWM frequency, $f_{s}$ & $20[\mathrm{kHz}]$ \\
Output filter inductance, $L$ & $1000[\mu \mathrm{H}]$ \\
Output filter capacitance, $C$ & $1000[\mu \mathrm{F}]$ \\
Load resistance, $R$ & $10[\Omega]$ \\
Operating duty cycle, $D$ & 0.08 \\
Input voltage, $V_{s}$ & $25[\mathrm{~V}]$ \\
Compensator & $R_{f}=5[\mathrm{k} \Omega], R_{l}=1[\mathrm{k} \Omega]$ \\
Current sensing resistance, $R_{s}$ & $0.2[\Omega]$ \\
Peak-to-peak voltage of ramp signal, $V_{m}$ & $1.7[\mathrm{~V}]$ \\
\hline
\end{tabular}




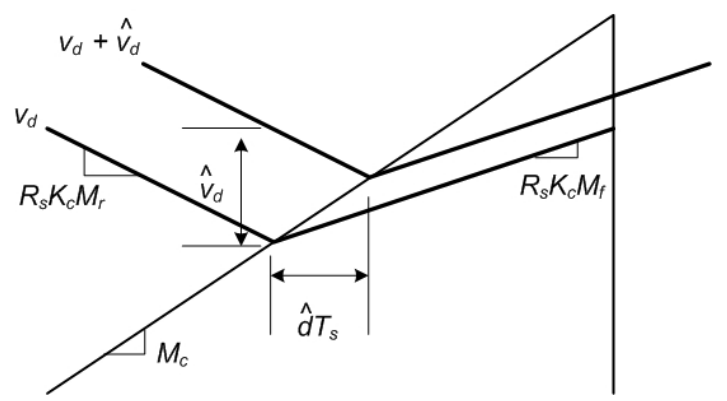

Fig. 4. Waveforms of PWM modulator.
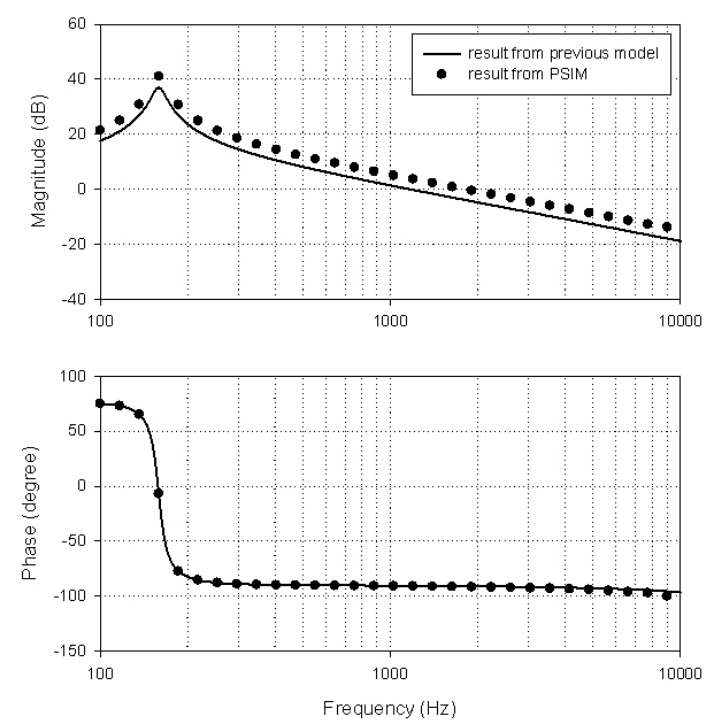

Fig. 5. Prediction results of the current loop gain from the conventional and PSIM models.

\section{SAmpler Model for P-Type Average Current MODE CONTROL}

In order to increase the prediction accuracy of the small signal model, we propose a sampler model for P-type average current mode control as shown in Fig. 6. In the proposed sampler model, the gain $T_{s}$ in PI-type control has been replaced with a zero-order holder ( $\mathrm{ZOH})$.

The sampler model can be simply obtained from Fig. 6 as was done with PI-type control. From the model structure shown in Fig. 6, the sampled quantity of the inductor current can be obtained as

$$
\hat{i}=\frac{F_{m} G_{Z O H}(s) G(s)}{1+R_{s} F_{m}\left[G_{Z O H}(s) K_{c} G(s)\right]^{*}}\left[\hat{i}_{c}^{*}+\left(K_{c} \hat{i}_{c}\right)^{*}\right]
$$

where the transfer function of the zero-order holder $G_{\mathrm{ZOH}}(s)$ is given as

$$
G_{Z O H}(s)=\frac{1-e^{-s T_{s}}}{s} .
$$

With the assumption of a slowly varying current command signal, we can get the following relation

$$
G_{\mathrm{ZOH}}(s) i_{c}^{*} \cong i_{c} \text {. }
$$

With (10), equation (8) could be rewritten as

$$
\hat{i}=\frac{F_{m}\left[1+K_{c}\right] G(s)}{1+R_{s} F_{m} T_{s}\left[K_{c} G(s)\right]^{*}} \hat{i}_{c} .
$$

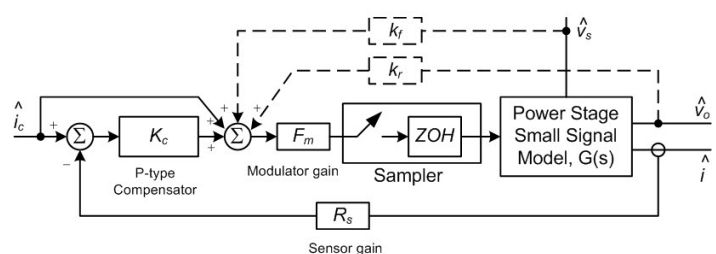

Fig. 6. Proposed small signal model structure for the P-type average current mode control.
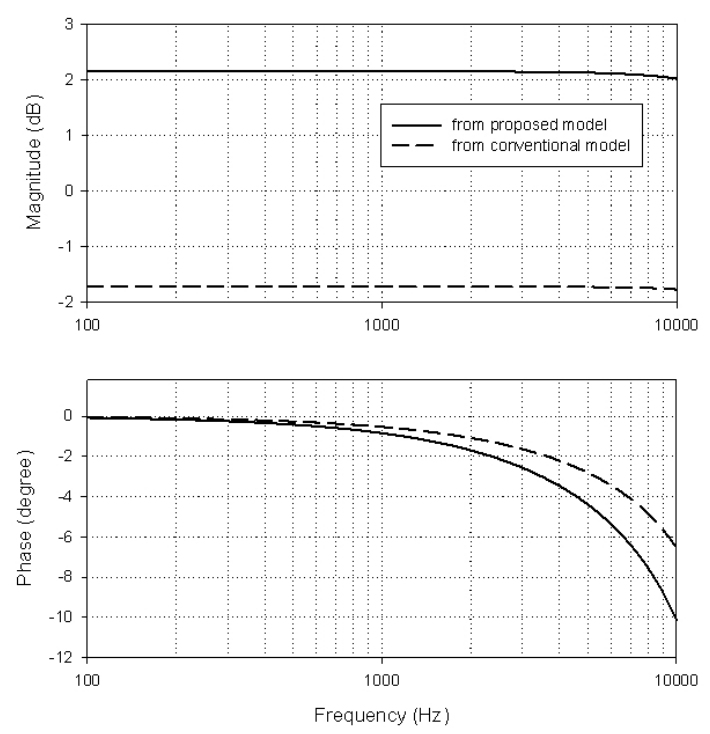

Fig. 7. Magnitude and phase responses for the conventional and proposed sampler models.

Denoting the equivalent expression of the sampler as $H_{s}(s)$, the current loop transfer function, $T_{i}(s)$, from the modeling structure shown in Fig. 6 is obtaine as

$$
T_{i}(s)=\frac{\hat{i}}{\hat{i}_{c}}=\frac{F_{m} G(s)\left[1+K_{c}\right] H_{s}(s)}{1+R_{s} F_{m} G(s) K_{c} H_{s}(s)} .
$$

From equations (11) and (12), the equivalent sampler gain is given as

$$
H_{s}(s)=\frac{1}{1+R_{s} F_{m}\left[\frac{1-e^{-s T_{s}}}{s} K_{c} G(s)\right]^{*}-R_{s} F_{m} K_{c} G(s)} .
$$

It is noted that the sampler gain obtained for P-type average current mode control is different from that for PI-type control. The frequency response for these two sampler gains are compared in Fig. 7. As can be seen in this figure, the proposed sampler model has a higher gain and a large phase delay near half of the switching frequency, which results in an increase in the prediction accuracy of the frequency response.

We could simplify the sampler model by manipulating the sampled quantities [8], [9] and using a Pade approximation defined as

$$
e^{-s T_{s}}=\frac{1-\frac{\pi}{\omega_{s}} s+\frac{4}{\omega_{s}^{2}} s^{2}}{1+\frac{\pi}{\omega_{s}} s+\frac{4}{\omega_{s}^{2}} s^{2}} .
$$

This approximation is known to be an accurate one for the frequency region below half of the switching frequency. 
The simplified sampler model for P-type average current mode control can be rewritten as

$$
H_{s}(s)=\frac{1}{\frac{\alpha T_{s}}{\pi^{2}} s+1-\frac{\alpha}{2}}
$$

where $\alpha=R_{s} F_{m} K_{c} T_{s}\left(M_{r}+M_{f}\right)$. It is noted from (15) that the sampler gain of P-type average current mode control is the same as that of peak current mode control except for the compensator gain $K_{c}$. The stability condition of the current loop gain can be examined using (15), and we can see that the current loop is stable when $\alpha$ is less than 2. And for stable operation of the PWM modulator, the following equation is required

$$
R_{s} K_{c} M_{f}<M_{c}
$$

From this condition, the inequality for $\alpha$ can be written as

$$
\alpha<\frac{1}{M_{r}+M_{f}} .
$$

In normal converter operation, the right hand side of equation (17) is less than 2. Thus, P-type average current mode control is always stable when (16) is satisfied.

\section{Application to the PEAK CURREnt MOde CONTROL}

In this section, we apply the proposed modeling to peak current mode control for comparison with the existing model. As noted in a previous section, the sampler model for peak current mode control can be obtained as (15) from (13) with a different modulator gain and $\mathrm{P}$ gain. The modulator gain, $F_{m}$ and the $\mathrm{P}$ gain, $K_{c}$ for peak current mode control could be given as

$$
\begin{aligned}
F_{m} & =\frac{\hat{d}}{\hat{v}_{d}}=\frac{1}{\left(M_{c}+R_{s} M_{r}\right) T_{s}} \\
K_{c} & =1
\end{aligned}
$$

where $M_{c}$ is the slope of the external stabilizing ramp signal. The sampler model introduces an additional pole into the current loop in the low frequency model. This result coincides with that given by the model in [6]. Even though the modulator gain is different from that of [6], the location of the additional pole and the frequency response of the current loop gain obtained from the proposed model are exactly the same with those from [6]. Table III shows some parameters associated with the sampler model for both the proposed and the existing models. In this table, to make it coincide with the existing model [6], the current sensing gain is assumed to be 1 and the current loop gain as

$$
T_{c s}(s)=\frac{\omega_{c}}{s\left(1+s / \omega_{p}\right)} .
$$

\section{VALidATIONS}

In this section, the sampler model obtained in this paper is validated by comparing it with a PSIM simulation. Fig. 8 shows the circuit diagram used in the PSIM simulation. The AC sweep is used to obtain the current loop gain of the PWM converter. In order to increase the accuracy of the simulation, the amplitude of the perturbed signal, $V_{\text {sweep }}$ is
TABLE III

COMPARISONS OF PROPOSED SAMPLER MODEL WITH EXISTING PLACEMODEL FOR PEAK CURRENT MODE CONTROL

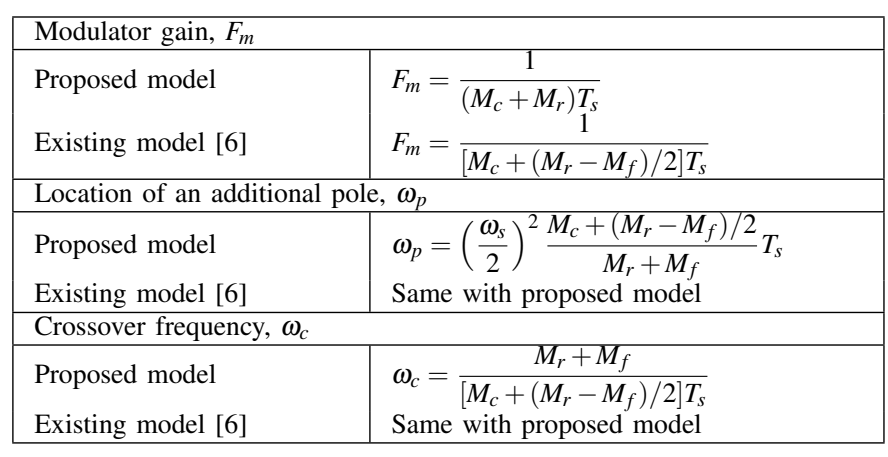

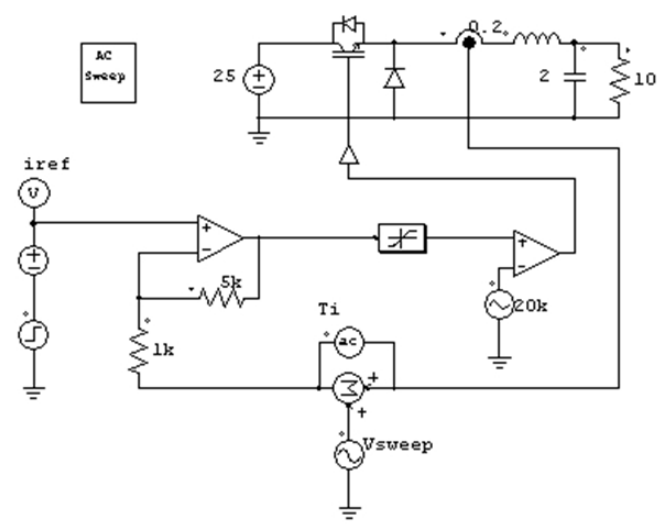

Fig. 8. PSIM simulation model to get the frequency response of the example converter.

required to be small enough when compared to the feedback signal. In our simulation study, we chose the amplitude of the perturbed signal to be 0.0028 , which is less than $1 / 10$ of the feedback signal. Fig. 9 shows the frequency response for three different models. The proposed model can predict the magnitude and phase response almost exactly the same as with the PSIM result when compared with the conventional model. Fig. 10 shows the enlarged phase response near half of the switching frequency. The phase delay near half of the switching frequency is also well predicted by the proposed model.

From the above results, it is found that different modeling approaches are required for different types of compensators to obtain an accurate sampler model.

\section{CONCLUSIONS}

This paper proposes a sampler model for P-type average current mode control. It is found that the sampler model obtained for PI-type control is not as accurate for P-type control as it is for PI-type control. With the proposed model, the prediction accuracy of the frequency response for the current loop is improved. This sampler model can also be applied to peak current mode control which might be considered as one kind of P-type control. The proposed model is applied to an example converter, and then the good accuracy of the proposed model is verified by comparing it with the result of the circuit level simulator, PSIM. 

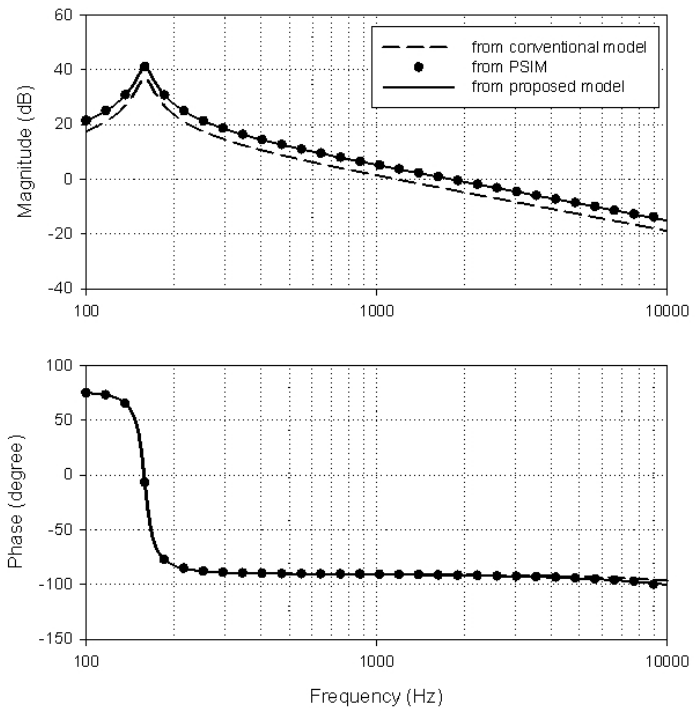

Fig. 9. Frequency responses for the proposed model, conventional model and PSIM model

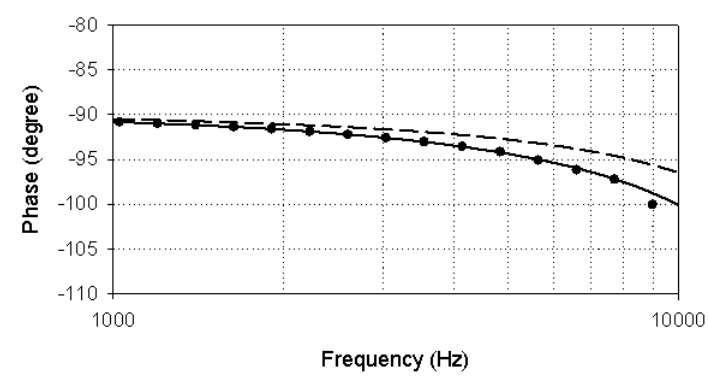

Fig. 10. Enlarged phase responses

\section{REFERENCES}

[1] L. H. Dixon, Average Current-Mode Control of Switching Power Supplies, Unitrode Power Supply Design Seminar Manual, 1990.
[2] T. Suntio, J. Lempinen, I. Gadoura and K. Zenger, "Dynamic effects of inductor current ripple in average current mode control," IEEE PESC Conf. Rec., pp. 1259-1264, 2001.

[3] J. Sun and R. M. Bass, "Modeling and practical design issues for average current control," IEEE APEC Record, pp. 980-986, 1999

[4] W. Tang, F. C. Lee, and R. B. Ridley, "Small-signal modeling of average current mode control," IEEE Trans. on Power Electronics, Vol. 8, No. 2, pp. 112-119, Apr. 1993.

[5] R. B. Ridley, "A new, continuous-time model for current-mode control," IEEE Trans. on Power Electronics, Vol. 6, No. 2, pp. 271-280, Apr. 1991.

[6] F. D. Tan and R. D. Middlebrook, "A unified model for currentprogrammed converters," IEEE Trans. on Power Electronics, Vol. 10, No. 4, pp. 397-408, Jul. 1995.

[7] Y.-S. Jung, "Modeling and design of average current mode control," Trans of KIPE, Vol. 10, No. 4, pp. 347-355, Aug. 2005.

[8] R. C. Dorf, Modern Control Systems, fifth edition, Addison Wesley.

[9] G. F. Franklin, J. D. Powell, and A. Emami-Naeini, Feedback Control of Dynamic Systems, 4th edition, Pearson \& Prentice Hall, 2002.

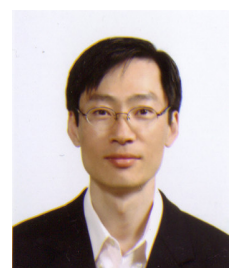

Young-Seok Jung received his B.S., M.S. and Ph.D. in Electrical Engineering from the Korea Advanced Institute of Science and Technology (KAIST), Daejon, Korea, in 1992, 1994, and 1999, respectively. He worked as a Senior Researcher for Hyundai Autonet, Powertrain Team, from 1999 to 2002. Since 2002, he has been with the Department of Mechanical and Automotive Engineering at Pukyong National University, Korea, where he is now an Associate Professor. His research interests are in the areas of power converters and variable speed motor drives. Dr. Jung is a member of the Korean Institute of Power Electronics (KIPE).

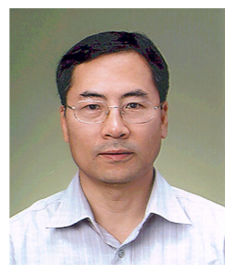

Marn-Go Kim received his B.S. in Electrical Engineering from Kyungpook National University in 1986, and his M.S. and Ph.D. in Electrical Engineering from the Korea Advanced Institute of Science and Technology in 1988 and 1991, respectively. From 1991 to 1994, he was at the Korea Telecom Research Center, where he worked in the research of telecom power systems such as uninterruptible power supplies, DC/DC converters, and distributed power systems. Since 1995, he has been with the Department of Control and Instrumentation Engineering, Pykyong National University, where he is now a Professor. His research interests include modeling, analysis, and control of resonant converters, power semiconductor circuits, and soft switching converters. Dr. Kim is a member of KIPE and KIEE. 